Journal for ImmunoTherapy of Cancer

\title{
VEGFR-2 redirected CAR-T cells are functionally impaired by soluble VEGF-A competition for receptor binding
}

\author{
Evripidis Lanitis (D) , ${ }^{1}$ Paris Kosti (D) , ${ }^{1}$ Catherine Ronet, ${ }^{1}$ Elisabetta Cribioli, ${ }^{1}$ \\ Giorgia Rota, ${ }^{1}$ Aodrenn Spill, ${ }^{1}$ Patrick Reichenbach, ${ }^{1}$ Vincent Zoete, ${ }^{2}$ \\ Denarda Dangaj Laniti, ${ }^{1}$ George Coukos, ${ }^{1}$ Melita Irving (D) ${ }^{1}$
}

To cite: Lanitis E, Kosti $P$, Ronet C, et al. VEGFR-2 redirected CAR-T cells are functionally impaired by soluble VEGF-A competition for receptor binding. Journal for ImmunoTherapy of Cancer 2021;9:e002151. doi:10.1136/ jitc-2020-002151

- Additional supplemental material is published online only. To view, please visit the journal online (http://dx.doi.org/10. 1136/jitc-2020-002151).

$\mathrm{GC}$ and $\mathrm{Ml}$ contributed equally. Accepted 07 June 2021

Check for updates

(C) Author(s) (or their employer(s)) 2021. Re-use permitted under CC BY-NC. No commercial re-use. See rights and permissions. Published by BMJ.

For numbered affiliations see end of article.

\section{Correspondence to \\ Melita Irving;}

melita.irving@unil.ch

George Coukos; george.coukos@chuv.ch

Evripidis Lanitis; evripidis.lanitis@unil.ch

\section{ABSTRACT}

Background The adoptive transfer of chimeric antigen receptor (CAR)-T cells has emerged as a potent immunotherapy against some hematological malignancies but not yet for epithelial-derived solid tumors. One critical issue is the paucity of broadly expressed solid tumor antigens (TAs), and another is the presence of suppressive mechanisms in the tumor microenvironment (TME) that can impair CAR-T cell homing, extravasation and effector functions. TAs expressed by endothelial cells of the tumor vasculature are of clinical interest for CAR therapy because of their genomic stability and accessibility to circulating T cells, as well as their expression across multiple tumor types. In this study, we sought to explore limitations to the efficacy of second-generation (2G) murine CAR-T cells redirected against the vascular endothelial growth factor receptor-2 (VEGFR-2) with the well-characterized singlechain variable fragment DC101.

Methods Primary murine T cells were retrovirally transduced to express a $2 \mathrm{G}$ anti-VEGFR-2-CAR, and the in vitro binding to VEGFR-2, as well as reactivity against TA-expressing cells, was evaluated in the absence versus presence of exogenous VEGF-A. The CAR-T cells were further tested in vivo for tumor control alone and in combination with anti-VEGF-A antibody. Finally, we performed ex vivo phenotypic analyses of tumor-infiltrating CAR-T cells for the two treatment groups.

Results In line with previous reports, we observed poor control of B16 melanoma by the $2 \mathrm{G}$ anti-VEGFR-2 CAR-T cells as a monotherapy. We further showed that VEGFR-2 is not downregulated by B16 melanoma tumors post treatment, but that its soluble ligand VEGF-A is upregulated and furthermore competes in vitro with the CAR-T cells for binding to VEGFR-2. This competition resulted in impaired CAR-T cell adhesion and effector function in vitro that could be restored in the presence of anti-VEGF-A antibody. Finally, we demonstrated that coadministration of anti-VEGF-A antibody in vivo promoted CAR-T cell persistence and tumor control and was associated with reduced frequencies of PD- $1^{+}$Ki67 ${ }^{-}$and LAG-3 $3^{+}$Ki67- CAR-T cells in the TME. Conclusions This study represents the first example of impaired function of a vasculature-targeted CAR by an angiogenic ligand and rationalizes the use of combinatorial therapies that target the tumor vasculature and augment CAR-T cell effector function.

\section{BACKGROUND}

Unprecedented responses of some advanced treatment-refractory hematological malignancies to CD19-targeted chimeric antigen receptor (CAR)-T cells led to rapid regulatory approvals and accelerated efforts in the field of $\mathrm{T}$ cell engineering for cancer immunotherapy. ${ }^{1-3}$ To date, however, limited clinical benefit has been reported for CAR-T cell treatment of epithelial-derived solid tumors. ${ }^{4}$ A major challenge is the identification of solid tumor antigens (TAs) that are broadly expressed on tumors and that do not run the risk of on-target but off-tumor toxicity. ${ }^{5}$ Indeed, while CD19 is mostly B-cell restricted, there are few solid TAs that are not also found on healthy tissue(s). ${ }^{6}$ Limited T cell homing is another obstacle, along with barriers to transendothelial migration of $\mathrm{T}$ cells across blood vessels into the tumor bed. ${ }^{7}$ In addition, a range of immunosuppressive factors such as programmed cell death ligand-1 (PDL1) can be upregulated in the tumor microenvironment (TME). ${ }^{8}$ Rationally designed combinatorial therapies and co-engineering strategies offer potential to bolster CAR therapy of solid tumors through TME reprogramming or/and direct augmentation of $\mathrm{T}$ cell function. ${ }^{3910}$

Tumors are reliant on a vasculature system for the delivery of nutrients and oxygen as well as the removal of metabolic waste, and they induce the formation of new blood vessels (ie, angiogenesis) in order to sustain their increasing metabolic needs as they grow. ${ }^{11}$ Angiogenesis is achieved by the release of proangiogenic growth factors, including vascular endothelial growth factor (VEGF) and basic fibroblast growth factor (bFGF). ${ }^{81314}$ A variety of anti-angiogenic therapies are used in the clinical management of 
cancer ${ }^{15}$; however, drug-induced resistance is problematic. ${ }^{16}{ }^{17}$ Vasculature-targeted therapies, mostly in the form of antibodies and kinase inhibitors, typically function by neutralizing growth factors or blocking their receptors, and they may promote vessel normalization ${ }^{18}$ to support immune cell infiltration and allow synergy with immunotherapy and other treatments such as radiotherapy and chemotherapy (reviewed in a previous work ${ }^{8}$ ). An alternative approach is the use of vascular disrupting agents (VDAs) to damage the established tumor (neo) endothelium and thereby cause tumor necrosis. ${ }^{19}$ VDAs, however, typically fail on their own because tumor adjacent to healthy tissue is supplied by its normal vasculature, thus enabling the tumor rim to rapidly regrow. ${ }^{19}$

The tumor vasculature is an appealing target for CAR-T cell therapy. ${ }^{20}$ Indeed, TAs expressed by endothelial cells of tumor blood vessels are more stably and homogeneously expressed in comparison to those found on tumors cells which typically have lower genomic stability (i.e., can be downregulated), and they are broadly shared across cancer types. ${ }^{2122}$ Moreover, targets within the vasculature compartment are readily accessible to circulating CAR-T cells. ${ }^{23}$ An important advantage of CAR-T cells in comparison to small molecules or antibody treatment is their longevity as they can establish memory in patients. ${ }^{24}$ In addition, CAR-T cells may induce epitope spreading and mobilize endogenous immunity. ${ }^{25}$ As such, CARs have been developed against a range of vasculature TAs including VEGFR-1, ${ }^{26}$ VEGFR-2, ${ }^{27-32}$ (neo)endotheliumassociated integrins, ${ }^{33}$ EIIIB domain containing fibronectin splice variant, ${ }^{34}$ NKG2D,${ }^{35}$ prostate-specific membrane antigen ${ }^{36}$ and tumor endothelial marker $8 .{ }^{37}$

Previous studies have demonstrated limited in vivo efficacy of tumor vasculature-targeted murine anti-VEGFR-2 CAR-T cells comprising the well-characterized singlechain variable fragment $(\mathrm{scFv})$ DC101 unless they are coadministered with high doses of interleukin (IL)-2, the CAR-T cells are co-engineered to express IL-12, or tumor-reactive $\mathrm{T}$ cells are cotransferred..$^{29}{ }^{38}$ In line with these studies, we recently demonstrated that the coexpression of murine IL-15 improves cellular fitness, reprograms the TME and augments tumor control by anti-VEGFR-2 CAR-T cells. ${ }^{40}$ Here we sought to explore limitations to the efficacy of anti-VEGFR-2 CAR-T cells as a monotherapy. Indeed, previous work has revealed that barriers within the tumor vasculature, such as the upregulation of FasL $\mathrm{L}^{41}$ and the aberrant expression of adhesion molecules, ${ }^{42}$ can attenuate T-cell function (reviewed in a previous work ${ }^{10}$ ). In this study, we noticed upregulation of VEGF-A following anti-VEGFR-2 CAR-T cell transfer and questioned whether this could impair endothelial cell adhesion as has been reported for $\mathrm{T}$ cells. ${ }^{42}$ While we did not observe changes to adhesion molecule expression levels on target cells in the presence of VEGF-A, we revealed physical competition for CAR-T cell binding to VEGFR-2 in vitro. Notably, we demonstrated rescue of anti-VEGFR-2 CAR-T cell function, both in vitro and in vivo, upon coadministration of anti-VEGF-A antibody.
Taken together, our findings support the use of combinatorial treatments targeting the tumor vasculature with CAR therapy.

\section{MATERIALS AND METHODS Cell culture}

The murine brain endothelioma cell line bEnd3 and the murine immortalized heart endothelial cell line $\mathrm{H} 5 \mathrm{~V}$ were cultured in Dulbecco's Modified Eagle's Medium (DMEM)-Glutamax comprising $4500 \mathrm{mg} / \mathrm{L}$ glucose and $110 \mathrm{mg} / \mathrm{L}$ sodium pyruvate, and supplemented with $10 \%$ heat-inactivated fetal bovine serum (FBS; Gibco, Thermo Fisher Scientific), $100 \mathrm{U} / \mathrm{mL}$ penicillin and $100 \mu \mathrm{g} / \mathrm{mL}$ streptomycin sulfate. The melanoma cell line B16-F10 was grown as a monolayer in DMEMGlutamax supplemented with $10 \%$ FBS, $100 \mathrm{U} / \mathrm{mL}$ penicillin and $100 \mu \mathrm{g} / \mathrm{mL}$ streptomycin sulfate. Cells were passaged twice weekly to be maintained under exponential growth conditions and were routinely tested for mycoplasma contamination. The Phoenix Eco retroviral ecotropic packaging cell line, derived from immortalized normal human embryonic kidney cells, was maintained in RPMI 1640-Glutamax medium supplemented with $10 \% \mathrm{FBS}, 100 \mathrm{U} / \mathrm{mL}$ penicillin and $100 \mu \mathrm{g} / \mathrm{mL}$ streptomycin sulfate. Primary murine T cells were cultured in RPMI 1640-Glutamax medium supplemented with $10 \%$ FBS, $100 \mathrm{U} / \mathrm{mL}$ penicillin, $100 \mu \mathrm{g} / \mathrm{mL}$ streptomycin sulfate, $1 \mathrm{mM}$ sodium pyruvate, $50 \mu \mathrm{M} \beta$-mercaptoethanol and $10 \mathrm{mM}$ non-essential amino acids (referred to as murine T-cell culture medium). T cells were activated 24 hours prior to transduction in murine T-cell culture medium supplemented with $50 \mathrm{IU} / \mathrm{mL}$ of human IL-2, and expanded from day 2 after transduction onwards in murine T-cell culture medium supplemented with $10 \mathrm{ng} /$ $\mathrm{ml}$ of both hIL-7 and hIL-15 as previously described. ${ }^{40}$

\section{Construction of CAR-encoding retroviral vectors}

The retroviral murine stem cell virus (MSCV)-based splice-gag vector (pMSGV), comprising the MSCV long terminal repeat, was used as the backbone for the antiVEGFR-2 CAR construct. The 2G anti-VEGFR-2 CAR (DC101-28z) construct comprising the anti-murine VEGFR-2 scFv DC101, the CD8 $\alpha$ hinge $(\mathrm{H})$ and transmembrane region, followed by the endodomains of CD28 and CD3ל, was kindly provided by Dr Steven A. Rosenberg (National Cancer Institute). As a control, a retroviral vector pMSGV encoding the marker Thy1.1 was built.

\section{Retrovirus production and murine $\mathrm{T}$ cell transduction}

Retrovirus was produced and primary murine $\mathrm{T}$ cells transduced and expanded as previously described. ${ }^{40}$ CAR-T cells were maintained at a cell density of $0.5-1 \times 10^{6}$ cells/mL to ensure optimal expansion. 


\section{Computational modeling of receptor-ligand binding}

The three-dimensional (3D) structure of the VEGFR2:DC101 complex was obtained by homology modeling using the experimental structure of ramucirumab in complex with domain 3 of VEGFR-2 (PDB ID $3 S 36^{43}$ ) at $3.2 \AA$ resolution. The sequence alignment between the DC101 and ramucirumab sequences was performed using the program MUSCLE. ${ }^{44}$ Based on this sequence alignment, 1000 structural models were generated using the MODELLER program ${ }^{45}$ and ranked according to the DOPE energy score. ${ }^{46}$ The top-ranked model according to DOPE was retained as the final model. Structural superimposition as well as molecular visualization and analysis were performed using the UCSF Chimera software. ${ }^{47}$

\section{Flow cytometry analysis}

VEGFR-2-28-z CAR expression was detected on $\mathrm{T}$ cells 7 days post-transduction by incubation with soluble recombinant murine (m)VEGFR-2-hIgG-Fc fusion protein (R\&D Systems) followed by staining with PE-labeled goat anti-IgG Fc (eBioscience). VEGFR-2 expression by murine endothelial cell lines was assessed by cell-surface staining with rat anti-VEGFR-2 antibody (Clone Avas12, BioLegend), and adhesion molecule surface expression was assessed with anti-ICAM-1 (Clone YN1/1.7.4, BioLegend) and anti-VCAM-1 (Clone MVCAM.A, BioLegend) antibodies. To discriminate dead cells, staining with 7-aminoactinomycin D (7-AAD, BioLegend) was performed. Data were acquired on a BD flow cytometer and analyzed using FlowJo software (Tree Star). For the ex vivo phenotypic analysis of CAR-T cells (from the spleens and tumors of differently treated mice), the following antibodies were used: CD45 (30F/11), CD3e (145-2C11), CD8 $\alpha$ (53-6.7), CD45.1 (A20), PD-1 (29F.1A12), LAG-3 (C9B7W), CTLA-4 (UC10-4B9) and Ki67 (SolA15). Antibodies were purchased from eBioscience and BioLegend or produced in-house from hybridomas by the flow cytometry platform. To exclude dead cells, the Live/dead fixable Aqua Dead cell stain kit was used according to the manufacturer's instructions (Molecular Probes, Life Technologies).

\section{Flow cytometry-based competitive binding assay}

CAR-T cells were incubated with $10 \mu \mathrm{g} / \mathrm{mL}$ recombinant mVEGFR-2-hIgG-Fc fusion protein (R\&D Systems) in the presence of control protein or soluble murine VEGF-A (VEGF-164, BioLegend), VEGF-C (BioLegend), or VEGF-D (R\&D Systems), at a VEGF/VEGFR-2 molar ratio of $3: 1$ in a total volume of $10 \mu \mathrm{L}$. Alternatively, VEGF-A was incubated with anti-VEGF-A antibody (Clone B20-4.1.1; a kind gift from Genentech, San Francisco, California, USA) at a VEGF-A:anti-VEGF-A antibody molar ratio of $1: 3$ for $30 \mathrm{~min}$ at $37^{\circ} \mathrm{C}$ prior its addition to CAR-T cells. The cells were then washed and stained to evaluate mVEGFR-2-hIgG-Fc binding levels on the CAR-T cells as described above.

\section{Adhesion assays}

Adhesion assays were performed using the Vybrant Cell Adhesion Assay Kit (Thermo Fisher Scientific) according to the manufacturer's instructions. Briefly, CAR-T cells were washed twice with phosphate-buffered saline (PBS) and then resuspended in RPMI at $5 \times 10^{6}$ cells $/ \mathrm{mL}$. Calcein AM stock solution was added at a final concentration of $5 \mu \mathrm{M}$. Samples were mixed well and incubated at $37^{\circ} \mathrm{C}$ for $30 \mathrm{~min}$. After labeling with calcein AM, CAR-T cells were washed twice and resuspended in plain RPMI at $1 \times 10^{6}$ cells $/ \mathrm{mL}$. Subsequently, $100 \mu \mathrm{L}$ of the calceinlabeled CAR-T cell suspension $\left(1 \times 10^{5}\right.$ cells unless otherwise indicated) was added to the prepared microplate wells containing confluent endothelial cell monolayers and incubated at $37^{\circ} \mathrm{C}$ for $90 \mathrm{~min}$. The plates were washed four times to remove non-adherent calcein-labeled cells and then $200 \mu \mathrm{L}$ of PBS was added to each well and fluorescence was measured. Prior to T-cell addition, target cells (where indicated) were exposed to bovine serum albumin (BSA) or VEGF-A or VEGF-A/anti-VEGF-A antibody complexes.

\section{Cytokine release assays}

Cytokine release was measured following: (1) exposure of T cells to 96-well plates coated with $1 \mu \mathrm{g} / \mathrm{mL}$ VEGFR-2 or control protein (BSA), (2) co-culture of $0.5 \times 10^{5} \mathrm{~T}$ cells with $0.5 \times 10^{5}$ target cells in 96 -well flat bottom plates, and (3) stimulation with anti- $(\alpha)$ CD3/CD28 dynabeads at a bead:cell ratio of 2:1. For the assays with immobilized antigen, the 96-well plates were treated with BSA, VEGF-A, VEGF-C, VEGF-D or VEGF-A/anti-VEGF-A antibody complexes at the indicated molar ratios for 1 hour at $37^{\circ} \mathrm{C}$ prior to T-cell addition. For the co-culture assays, the target cells were treated with $0.17 \mu \mathrm{g} / \mathrm{mL}$ VEGF-A or VEGF-A/VEGF-A antibody complexes at molar ratio 1:3 or BSA for 1 hour at $37^{\circ} \mathrm{C}$ prior to T-cell addition. For the assays of $\mathrm{T}$ cell stimulation with $\alpha \mathrm{CD} 3 / \mathrm{CD} 28$ dynabeads, VEGF-A, VEGF-C, or VEGF-D were added at a concentration of 0.17 or $0.5 \mu \mathrm{g} / \mathrm{mL}$. At $20-24$ hours post stimulation with immobilized antigen or target cells or $\alpha \mathrm{CD} 3 /$ CD28 dynabeads, the supernatants were assayed for the presence of interferon- $\gamma$ (IFN- $\gamma$ ) by commercial ELISA Kit (BioLegend). The levels of IL-2, tumor necrosis factor- $\alpha$ $(\mathrm{TNF}-\alpha)$ and macrophage inflammatory protein-1 alpha $(\mathrm{MIP}-1 \alpha)$ were quantified using the Cytokine Bead Array (CBA) as described by the manufacturer (BD Biosciences).

\section{Mouse strains}

CD45. $1^{+}$congenic C57BL/ 6 mice were bred in the Epalinges UNIL animal facility. Female CD $45.2^{+}$C57BL/ 6 mice aged 6-8 weeks were purchased from Harlan (Harlan, Netherlands). All in vivo experiments were conducted in accordance with approval from the Service of Consumer and Veterinary Affairs (SCAV) of the Canton of Vaud. 


\section{Adoptive CAR-T cell transfer in tumor-bearing mice}

B16 tumor cells were harvested with $0.05 \%$ trypsin, washed, and resuspended in PBS for injection. $1 \times 10^{5}$ tumor cells were injected subcutaneously in the flank of C57BL/6 mice, aged 8-12 weeks. Nine days later (average tumor volume $20-40 \mathrm{~mm}^{3}$ ), the mice received $5 \mathrm{~Gy}$ of sublethal total body irradiation and grouped ( $\mathrm{n} \geq 5$ mice/ group) for comparative average tumor volumes. On days 10 and 13 , the mice were treated with intravenous injection of $8 \times 10^{6} \mathrm{CD} 45.1^{+}$CAR-T cells or control Thy1.1transduced $\mathrm{T}$ cells. In some experiments, anti-VEGF-A antibody $(40 \mu \mathrm{g})$ was coadministered on the days of ACT and then every 2-4days. Mice were carefully monitored and tumor length (L; greatest longitudinal measurement) and width (W; greatest transverse measurement) were measured by caliper every 2-3 days. Tumor volumes (V) were calculated using the formula: $\mathrm{V}=\left(\mathrm{L} \times \mathrm{W}^{2}\right) / 2$. The average tumor volumes/group are plotted \pm SEM. Mice were sacrificed once tumors reached $1000 \mathrm{~mm}^{3}$, or if they became distressed or moribund, according to regulations.

\section{Immunofluorescence staining of B16 tumors post-CAR-T cell treatment}

Tumors were embedded and frozen in Tissue-Tek OCT (Sakura Finetek). Cryostat sections $(5-8 \mu \mathrm{m})$ were collected on Superfrost/Plus glass slides (Thermo Fisher Scientific) and then air-dried overnight before being stored at $-20^{\circ} \mathrm{C}$ until further analysis. The day of the staining slides were warmed, rehydrated with PBS for 5 min at room temperature (RT), fixed in $10 \%$ neutral buffered formalin for $10 \mathrm{~min}$ at $4^{\circ} \mathrm{C}$ and washed three times in PBS. Sections were permeabilized with $0.5 \%$ Triton X-100 in PBS for $10 \mathrm{~min}$ at RT, washed $(4 \times 5 \mathrm{~min}$ at RT) and blocked using PBS with 1\% BSA and 5\% FBS for 2 hours at RT. Immunofluorescence primary stainings were performed with anti-CD31 (Clone MEC 13.3, BD Biosciences) and anti-VEGFR-2 (R\&D Systems, Catalog \# AF644) antibodies diluted in blocking solution and incubated on sections overnight at $4^{\circ} \mathrm{C}$. Secondary staining was then performed for 1 hour at RT using donkey anti-rat IgG-Alexa488 and donkey anti-goat-Alexa 568 (Invitrogen). All slides were then stained with DAPI (SigmaAldrich, St Louis, Missouri, USA) and mounted using DABCO solution (Sigma-Aldrich). Images were acquired on a Zeiss AxioVision microscope with an AxioCam and processed using AxioVision software and Image J. To analyze the vessel presence in the tumor area as well as the CD31 MFI, the staining was evaluated on three to six different areas of each tumor. A similar procedure was performed for the VEGFR-2 staining and analysis.

\section{Quantitative PCR}

RNA and cDNA preparation from dissociated tumors and relative quantification of the DC101 scFv using fast SYBR Green-based reagents (Applied Biosystems) was performed as previously described. ${ }^{40}$ The following primers were used for DC101 scFv: DC101-Forward: 5'-GCAACCCAAACTCCTCATCT-3';
5'-TATCATCAGCCTCCACAGGA-3'. The primers for GAPDH applied in the SYBR Green-based qPCR and used for normalization of the RNA levels were the following: GAPDH-Forward: 5'-AGGTCGGTGTGAACGGATTTG-3' and GAPDH-Reverse: 5'-TGTAGA CCATGTAGTTGAGGTCA-3'. Evaluation of the relative mRNA expression for VEGFR-2, VEGF-A, CD8 $\alpha$ and IFN- $\gamma$ (normalized to GAPDH) was performed using TaqMan-based reagents and primers (Applied Biosystems). Each sample was run in triplicate and data were acquired using the 7500 Fast Real-Time PCR System (Applied Biosystems).

\section{Determination of serum and tumor VEGF-A levels}

For murine VEGF-A protein quantification in the serum, blood was drawn on the day of ACT and placed in standard $1.5 \mathrm{~mL}$ Eppendorf tubes followed by incubation in a standing position at RT for $30 \mathrm{~min}$. The tubes were then centrifuged at $2000 \times \mathrm{g}$ for $10 \mathrm{~min}$ at $4^{\circ} \mathrm{C}$ to separate the clot from the supernatant (serum) and mVEGF-A was measured in the serum by ELISA (Mouse VEGF DuoSet ELISA, RD Systems) according to the manufacturer's instructions. For mVEGF-A quantification in the tumors, extracted tumors were smashed using glass potter tissue grinders (Thermo Fisher Scientific) in PBS buffer containing EDTA-free Protease Inhibitors (Thermo Fisher Scientific, Catalog \# A32965) and phenylmethylsulfonyl fluoride (PMSF; Roth, Catalog \# A32965) according to the manufacturer's instructions. The smashed tumors were then transferred to $1.5 \mathrm{~mL}$ Eppendorf tubes and centrifuged at $12,000 \times \mathrm{g}$ for $10 \mathrm{~min}$ at $4^{\circ} \mathrm{C}$. The resulting supernatants were then subjected to another centrifugation before quantifying the levels of mVEGF-A by ELISA.

\section{Evaluation of the in vivo phenotype of adoptively transferred CAR-T cells}

Spleens and tumors were harvested from the differently treated mice. Splenocytes were gently crushed through a $40 \mu \mathrm{m}$ cell strainer followed by RBC lysis. Tumor fragments were cut into pieces with scissors and then digested in RPMI supplemented with $200 \mu \mathrm{g} / \mathrm{mL}$ Liberase TL (Roche) and 5 units/mL DNase I (Sigma-Aldrich) for 1 hour at $37^{\circ} \mathrm{C}$ on a rocker, followed by passage through a $40 \mu \mathrm{m}$ cell strainer. The ex vivo CAR-T cell phenotype was assessed by flow cytometry using antibodies mentioned above.

\section{Statistical analysis}

All statistical analyses were performed using GraphPad Prism V.8 software. Analysis of differences between two groups was performed using an unpaired two-tailed Student's t-test. Statistical analyses of three or more groups were performed using a one-way analysis of variance (ANOVA) followed by Tukey's post hoc correction test. Statistical analysis of tumor growth curves was performed using a two-way repeated measures ANOVA followed by Newman-Keuls post hoc correction test. Significance levels are indicated with stars in the figures 
and are the following: ${ }^{*} \mathrm{p}<0.05,{ }^{*} \mathrm{p}<0.01, * * * \mathrm{p}<0.001$ and $* * * * \mathrm{p}<0.0001$.

\section{RESULTS \\ VEGFR-2 redirected murine CAR-T cells mediate poor B16 tumor control, but this is not due to target antigen loss}

In our study, we used a 2G CAR comprising the antiVEGFR-2 scFv DC101 ${ }^{29}$ fused to the hinge and transmembrane domain of $\mathrm{CD} 8 \alpha$, followed by the endodomains of CD28 and CD3ל (figure 1A). Primary murine T cells were efficiently engineered ( $>80 \%$ transduction, as described in a previous work ${ }^{40}$ ) with retrovirus encoding the CAR (or Thy1.1 for control $\mathrm{T}$ cells) as evaluated by soluble recombinant mVEGFR-2-hIgG-Fc fusion protein staining and flow cytometric analysis on day 7 post-transduction (figure 1B). The adoptive transfer of $8 \times 10^{6} \mathrm{CD} 45.1^{+}$ anti-VEGFR-2-CAR-T cells in lymphodepleted B16 melanoma tumor-bearing mice on days 10 and 13 post-tumor cell engraftment (in vivo treatment schematic shown in figure 1C) failed to control tumor growth (figure 1D). This is in line with previous studies showing poor responses with this $2 \mathrm{G}$ CAR as a monotherapy in the context of several tumor models. ${ }^{27-32}$

We first questioned whether TA loss may account for poor tumor control by the CAR-T cells. Immunofluorescent analysis of B16 tumors following adoptive cell transfer (ACT), however, showed that this was not the case as the distribution and density of CD31 and VEGFR-2 was similar in both CAR-T cell and control Thyl.1-T cell treatment groups (figure $1 \mathrm{E}-\mathrm{G}$ ). We further evaluated VEGF-A gene expression levels and observed a significant increase in the tumors of CAR-T cell versus control-T cell-treated mice, unlike VEGFR-2 gene expression which did not differ (figure 1H). Intrigued by this observation, coupled with the previous demonstration that VEGF-A can cause clustering defects of adhesion molecules, ${ }^{42}$ we next sought to evaluate if soluble VEGF-A could impair the adhesion and function of VEGFR-2 directed CAR-T cells against target cells.

\section{Competitive binding by VEGF-A impairs anti-VEGFR-2 CAR-T cell adhesion to target cells}

To evaluate changes to CAR-T cell adhesion in the presence of VEGF-A, we began by setting up an in vitro assay (as illustrated in figure 2A) in which fluorescently labeled $\mathrm{T}$ cells were deposited onto confluent monolayers of bEnd3 (VEGFR-2 ${ }^{+}$) or H5V (VEGFR-2') endothelial cells (figure 2B). Low levels of adhesion were observed for Thy1.1-T cells to both bEnd3 and H5V cells (figure 2C). In contrast, anti-VEGFR-2 CAR-T cells strongly bound to bEnd3 endothelial cells but poorly to $\mathrm{H} 5 \mathrm{~V}$ cells (figure $2 \mathrm{C}, \mathrm{D}$ ). A repetition of the assay for $1 \times 10^{5}$ CAR-T cell and Thyl.1-T cells revealed a significant drop in CAR-T cell adhesion to bEnd3 cells in the presence of soluble VEGF-A (figure 2E). However, we observed that bEnd3 exposure to VEGF-A did not change the expression levels of intracellular adhesion molecule-1
(ICAM-1), vascular cell adhesion molecule-1 (VCAM-1) and VEGFR-2 (figure 2F). While these observations did not preclude the possibility of clustering defects of adhesion molecules, we next sought to explore the possibility that soluble VEGF-A, a natural angiogenic ligand of VEGFR-2, interferes with target receptor binding by the DC101 scFv-based CAR.

We interrogated structural databases and identified the human anti-VEGFR-2 antibody ramucirumab, which has been demonstrated to sterically hinder VEGF-A engagement with VEGFR-2, ${ }^{43}$ as having sequence similarity to DC101; $65 \%$ homology for the heavy chain and $~ 58 \%$ for the light chain (figure $2 \mathrm{G}$, online supplemental figure S1). By homology modeling using ramucirumab in complex with domain (D) 3 of VEGFR-2 (PDB ID $3 S 36^{43}$ ), we predicted the VEGFR-2:DC101 structure (figure 2H) which we then superimposed on the structure of the human VEGFR-2:VEGF-A heterotetramer (PDB ID $\left.3 \mathrm{~V}^{4} \mathrm{~A}^{48}\right)$. Superimposing the predicted and experimental structures revealed that in this model DC101 binds to D2 and D3 of VEGFR-2 and into the groove between them, and that residues 51-IEDKSNNYFIS-61 of DC101 occupy the same space as VEGF-A (figure 2I,J). Hence, the computational modeling indicated that bound VEGF-A may sterically hinder DC101 scFv engagement with VEGFR-2.

\section{Soluble VEGF-A abrogates CAR-T cell binding to VEGFR-2 and attenuates effector function}

We next sought to experimentally test if soluble VEGF-A interferes with the DC101 scFv-based CAR binding to mVEGFR-2. We thus mixed anti-VEGFR-2 CAR-T cells with recombinant mVEGFR-2-hIgG-Fc that had been preincubated with VEGF-A or preassembled VEGF-A/anti-VEGF-A antibody complexes (figure 3A). As expected, the control Thy1.1-T cells did not bind to mVEGFR-2-hIgG-Fc under any conditions tested. Anti-VEGFR-2 CAR-T cells strongly bound mVEGFR-2-hIgG-Fc, but not if mVEGFR-2-hIgG-Fc had been preincubated with VEGF-A (figure 3B). In addition, anti-VEGFR-2 CAR-T cells mixed with VEGF-A/antiVEGF-A antibody complexes strongly bound to soluble mVEGFR-2-hIgG-Fc (figure 3B).

Next, as depicted in figure 3C, we evaluated antiVEGFR-2 CAR-T cell effector function against platecaptured target antigen (i.e., VEGFR-2) under the same conditions. Anti-VEGFR-2 CAR-T cells secreted high levels of IFN- $\gamma$ and IL-2 in the presence of target antigen (figure 3D and F), while cytokine production was attenuated in the presence of soluble VEGF-A but not in the presence of VEGF-A/anti-VEGF-A antibody complexes (figure $3 \mathrm{E}$ and $\mathrm{G}$ ). In contrast, we found that the other VEGFR-2 ligands, namely VEGF-C and VEGF-D, did not impair CAR-T cell binding with soluble mVEGFR2-hIgG-Fc (online supplemental figure S2A,B), nor did they attenuate CAR-T cell effector function (assessed by IFN- $\gamma$ production) as was observed for VEGF-A (online supplemental figure S2C). Finally, to address a direct impact of VEGF-A, VEGF-C or VEGF-D on effector function, CAR-T cells were activated with $\alpha \mathrm{CD} 3 / \mathrm{CD} 28$ beads 
A

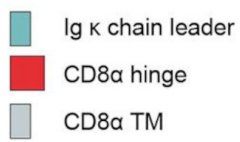

2G-CAR:
VEGFR-2-28z

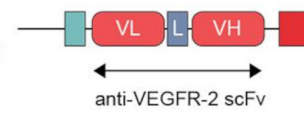

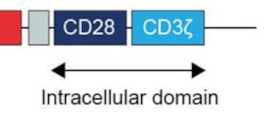

B

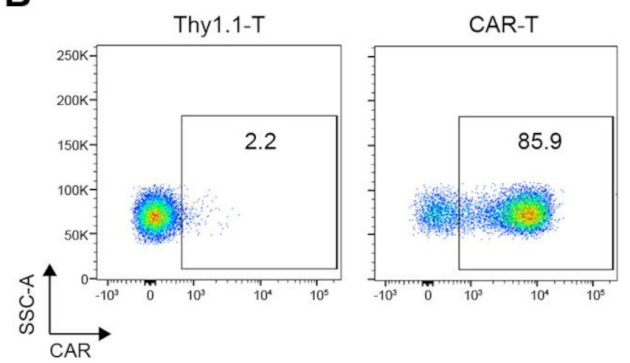

C

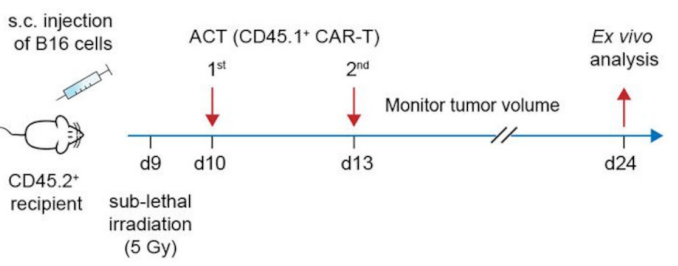

D

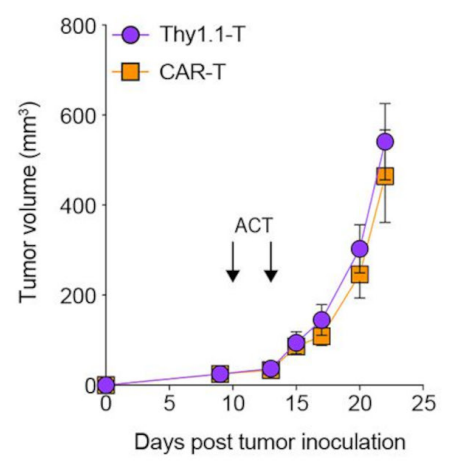

E

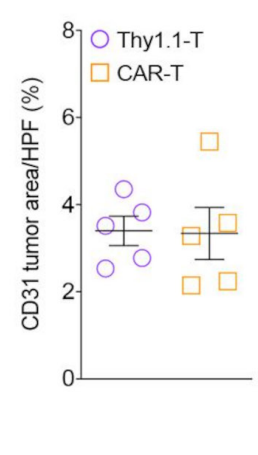

CD31

F

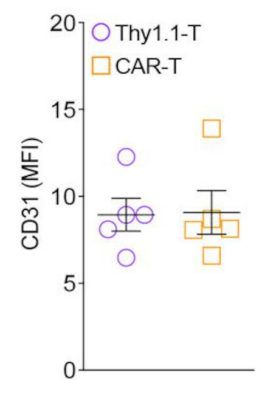

G

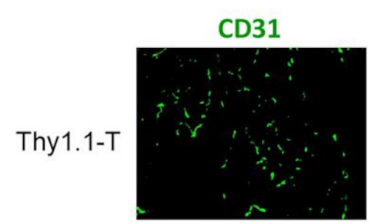

CAR-T

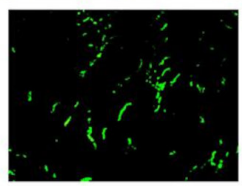

VEGFR-2
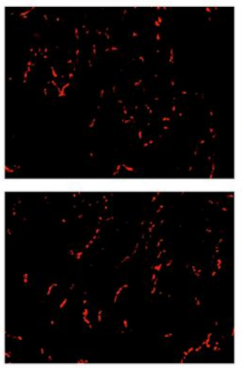

DAPI
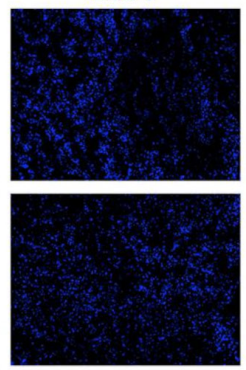

H

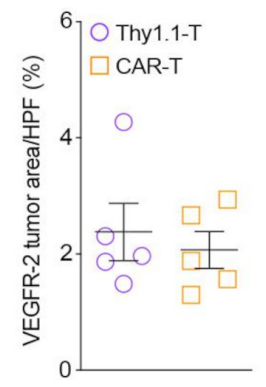

VEGFR-2
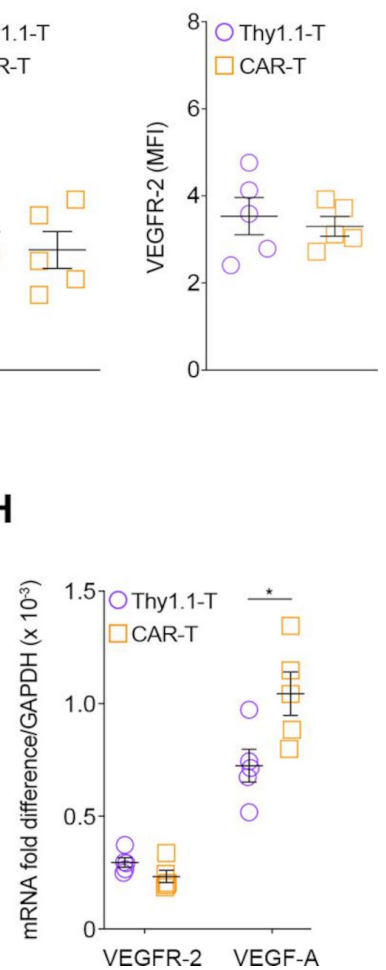

Figure 1 The inability of anti-VEGFR-2 CAR-T cells to control tumor growth cannot be attributed to target antigen loss. (A) Schematic depicts the design of the anti-VEGFR-2 CAR used in the study. The anti-VEGFR-2 CAR is comprised of the scFv DC101, a CD8 $\alpha$ hinge and transmembrane (TM) domain, followed by CD28 costimulatory and CD3 $\zeta$ signaling endodomains (DC101, $\alpha$ VEGFR-2 scFv; L, linker; VL, variable light chain; VH, variable heavy chain). (B) Representative flow cytometry plots showing surface detection of the anti-VEGFR-2 CAR on primary murine T cells following retroviral transduction. (C) Schematic of ACT study and ex vivo analysis. Arrows indicate days of T-cell infusion. (D) Assessment of B16 melanoma tumor growth control in mice treated with CAR-T cells or control Thy1.1-T cells. Results are expressed as mean tumor volume $\left(\mathrm{mm}^{3}{ }^{3} \mathrm{SEM}\right)$ with $n=6$ mice per group. (E-F) Immunofluorescence analysis to determine the percentage of the tumor area stained with antiCD31 (E, left) or anti-VEGFR-2 antibody ( $F$, left) and the fluorescent intensity of CD31 (E, right) or VEGFR-2 (F, right) staining in tumors from mice treated with Thy1.1-T cells or CAR-T cells. Each value shown in the graphs represents the mean of 5-7 independent HPF (high-power field) analyzed per mouse ( $n=5$ mice per group). (G) Representative immunofluorescent images of the vascular network analysis. Staining for CD31 (green), VEGFR-2 (red) and 4',6-diamidino-2-phenylindole (DAPI; blue). (H) Relative mRNA quantification of VEGFR-2 and VEGF-A in the tumors of all treatment groups. Results are presented as mean mRNA fold difference (relative to GAPDH) \pm SEM with $n=5$ mice per group. Statistical analysis was performed using a two-tailed unpaired Student's t-test; ${ }^{*} p<0.05$. CAR, chimeric antigen receptor; scFv, single-chain variable fragment; VEGF-A, vascular endothelial growth factor-A; VEGFR-2, vascular endothelial growth factor receptor-2. 
A
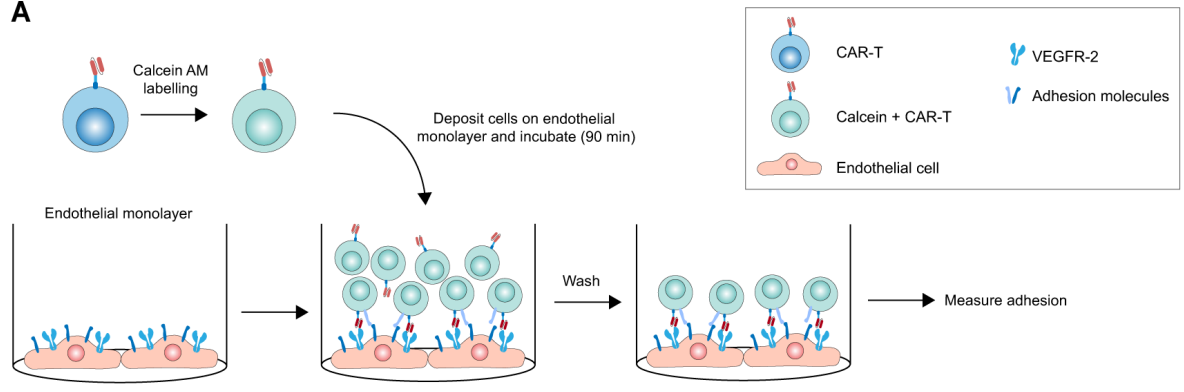

B

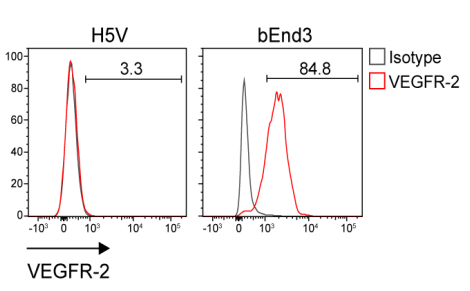

C

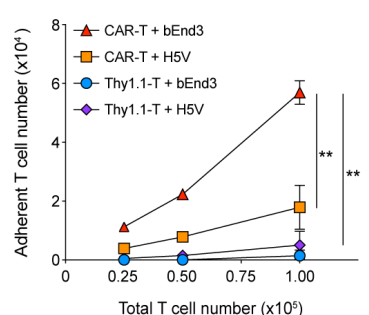

D

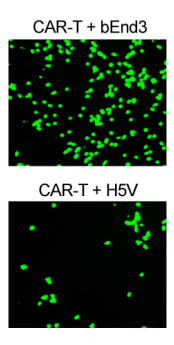

E

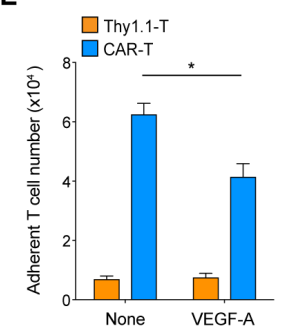

$\mathbf{F}$

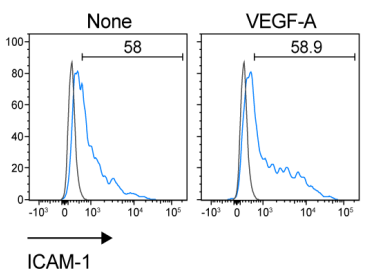

G

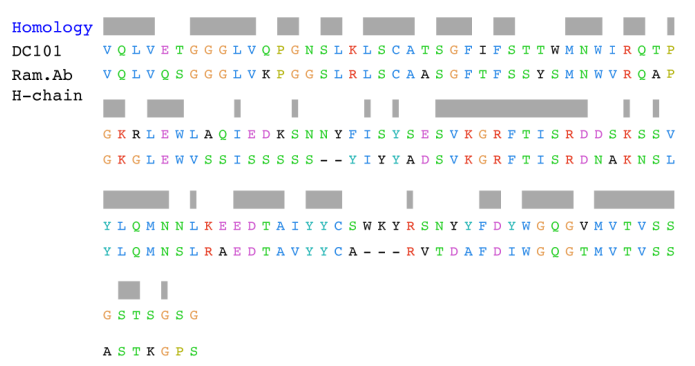

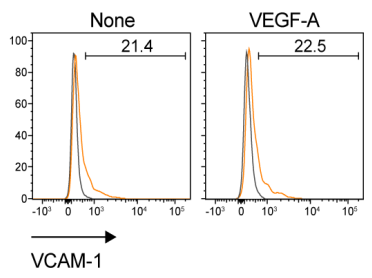

H

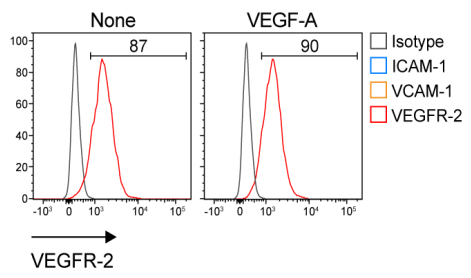

I
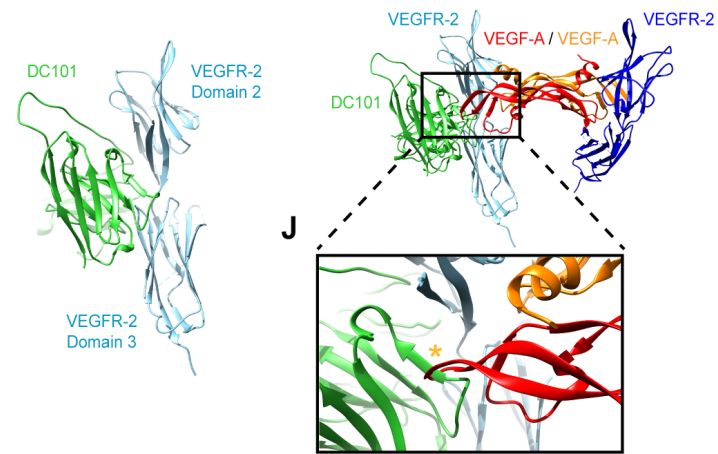

Figure 2 Soluble VEGF-A attenuates CAR-T cell adhesion to VEGFR-2 ${ }^{+}$endothelial cells. (A) Schematic demonstrating the adhesion assay. (B) Histograms showing VEGFR-2 (red line) expression by endothelial cell lines. Gray line represents the isotype control. (C) Adhesion as quantified by fluorescence of Thy1.1-T cells or CAR-T cells added at increasing numbers onto H5V or bEnd3 endothelial cells. Results are presented as mean number of adherent cells \pm SEM of duplicate wells with T cells pooled from $n=4$ mice. (D) Representative images from adhesion experiments $(\times 20$ magnification; green, Calcein AM-labeled CAR-T cells). (E) Quantification of the adhesion of CAR-T cells or Thy1.1-T cells to resting endothelial cells or endothelial cells pre-exposed to $100 \mathrm{ng} / \mathrm{mL}$ VEGF-A for 4.5 hours. Results are presented as the mean number of adherent cells \pm SEM of triplicate wells with $T$ cells pooled from $n=4$ mice. (F) Representative histograms indicating the expression of ICAM-1 (blue), VCAM-1 (orange), and VEGFR-2 (red) on resting endothelial cells or endothelial cells treated with 100 ng/mL VEGF-A. (G) Protein sequence alignment and homology of DC101 scFv with the heavy chain of ramucirumab. Gray rectangles represent regions of identical sequences. (H) Model structures of the DC101 in complex with VEGFR-2. DC101 and VEGFR-2 are shown in ribbon representation, colored in green and blue, respectively. (I) Superimposition of the modeled structure of the VEGFR2:DC101 complex, with the experimental 3D structure of the VEGFR-2:VEGF-A heterotetramer. All proteins are shown in ribbon representation, with the two monomers of VEGFR-2 colored in light and dark blue, and the two VEGF-A monomers colored in red and orange. (J) Same as in (I) but focused on the volume occupied by both DC101 and VEGF-A in their respective complexes. The virtual steric clash between DC101 and VEGF-A, close to the * symbol, indicates that DC101 and VEGF-A compete for VEGFR-2 binding. Statistical analysis in (C) was performed using a one-way ANOVA with Tukey post hoc correction test and in (E) using a two-tailed unpaired Student's t-test; ${ }^{*} \mathrm{p}<0.05$; ${ }^{* *} \mathrm{p}<0.01$. CAR, chimeric antigen receptor; ICAM-1, intracellular adhesion molecule-1; VCAM-1, vascular cell adhesion molecule-1; VEGF-A, vascular endothelial growth factor-A; VEGFR-2, vascular endothelial growth factor receptor-2. 
A
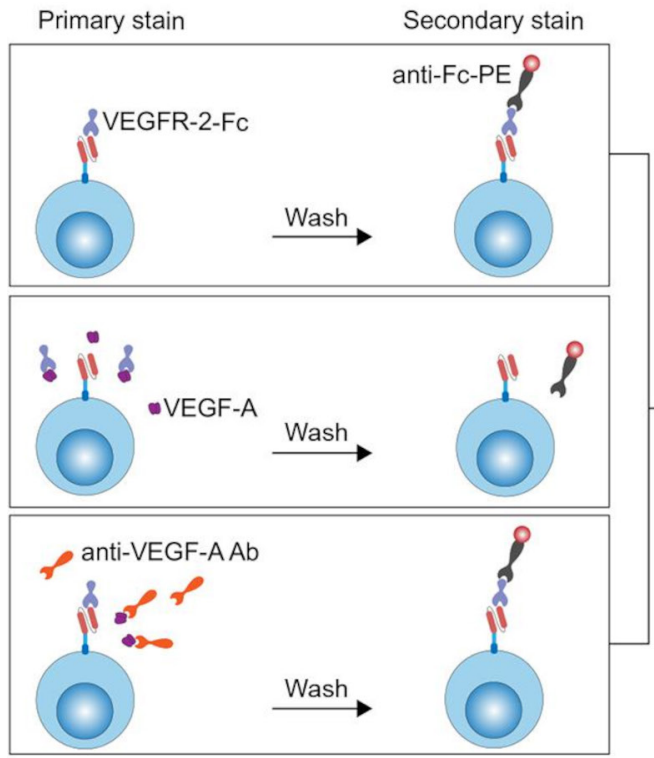

C

YVEGFR-2-FC

(1) Coat VEGFR-2

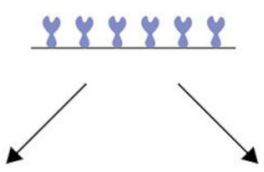

(2) + VEGF-A or VEGF-A + anti-VEGF-AAb

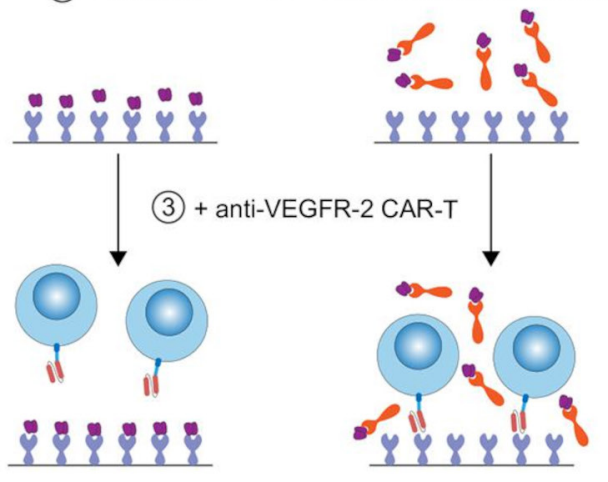

(4) Measure cytokines (20 hrs)
B

Thy1.1-T

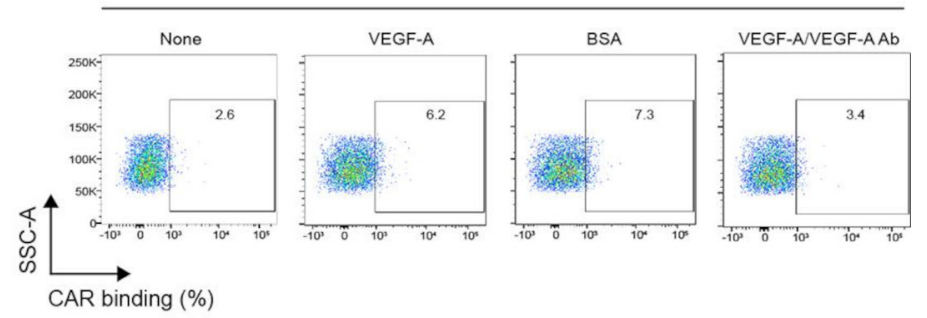

CAR-T

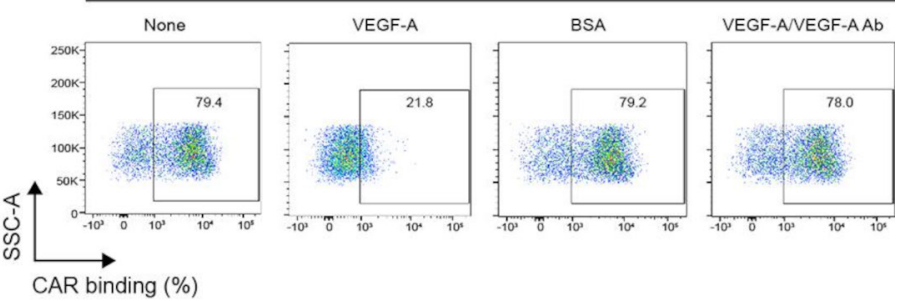

E
VEGF-A:VEGFR-2 $=3: 1$

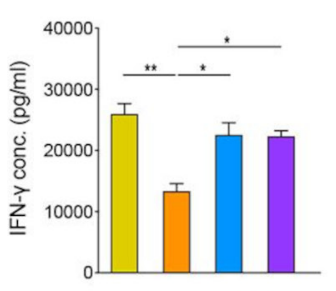

$\square$ BSA $\square$ VEGF-A $\square$ VEGF-A:VEGF-AAb (1:1) $\square$ VEGF-A:VEGF-AAb (1:3)

G

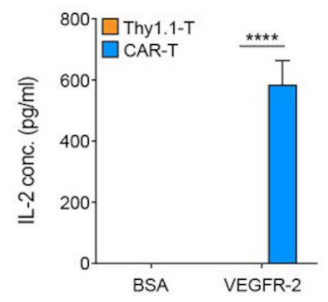

VEGF-A:VEGFR-2 = 1:1

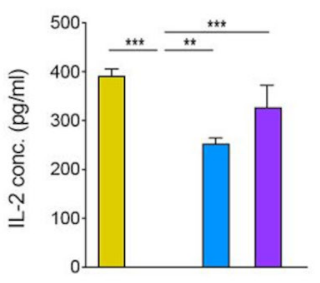

$\square$ BSA $\square$ VEGF-A $\square$ VEGF-A:VEGF-AAb (1:1) $\square$ VEGF-A:VEGF-AAb (1:3)

Figure 3 CAR binding to VEGFR-2 antigen and antigen-induced CAR-T cell effector function are impaired in the presence of soluble VEGF-A. (A) Schematic of the flow cytometry method used for assessing whether VEGF-A inhibits binding of the CAR to its target antigen. (B) Representative flow cytometry plots presenting the binding of CAR-T cells to mVEGFR-2-hlgGFc in the presence of VEGF-A (at a VEGF-A:VEGFR-2 molar ratio of 3:1) or VEGF-A/VEGF-A antibody (Ab) complexes (at a VEGF-A/VEGF-A Ab molar ratio of 1:3) or control protein bovine serum albumin (BSA). The staining was performed with $T$ cells pooled from $n=5$ mice. (C) Schematic of the method used for evaluating CAR-T cell reactivity against immobilized target antigen in the presence of VEGF-A or VEGF-ANVGF-A Ab complexes. (D-G) (D) and (F) graphs show the secretion of IFN- $\gamma$ and IL-2, respectively, by CAR-T cells vs Thy1.1-T cells in response to immobilized VEGFR-2 or BSA. (E) and (G) graphs show the secretion of IFN- $\gamma$ and IL-2, respectively, by CAR-T cells in response to immobilized VEGFR-2 in the presence of soluble BSA or VEGF-A or VEGF-ANEGF-A Ab complexes at the indicated molar ratios. Results in (D-G) are presented as the mean concentration $(\mathrm{pg} / \mathrm{mL}) \pm S E M$ of triplicate wells for IFN- $\gamma$ or IL-2 secreted by $T$ cells pooled from $n=4$ mice. Statistical analyses in (D) and (F) were performed using a two-tailed unpaired Student's t-test and in (E) and (G) using a one-way ANOVA with Tukey correction test; ${ }^{*} \mathrm{p}<0.05 ;{ }^{* *} \mathrm{p}<0.01$; ${ }^{\star * *} \mathrm{p}<0.001$; ${ }^{* * *} \mathrm{p}<0.0001$. CAR, chimeric antigen receptor; IFN- $\gamma$, interferon- $\gamma$; IL-2, interleukin-2; VEGF-A, vascular endothelial growth factor-A; VEGFR-2, vascular endothelial growth factor receptor-2. 
in the presence of supraphysiologic concentrations of the different molecules but there were no changes in IFN- $\gamma$, MIP- $1 \alpha$, TNF- $\alpha$ and IL-2 production (online supplemental figure S3A-D). Taken together, the computational and experimental data indicate that soluble VEGF-A sterically hinders DC101 scFv-based CAR-T cell engagement with VEGFR-2, thereby attenuating effector function.

\section{Coadministration of anti-VEGF-A antibody synergizes with anti-VEGFR-2 CAR-T cell transfer for enhanced tumor control}

Before exploring VEGF-A blockade in vivo, we sought to set up competition assays in the context of target endothelial cells. As depicted in figure 4A, target bEnd3 monolayers were exposed to soluble VEGF-A, VEGF-A/ anti-VEGF-A antibody complexes, or control protein, prior to the addition of anti-VEGFR-2 CAR T cells (or control Thy1.1-T cells). As was previously observed in the context of plate-captured target antigen, both adhesion (figure 4B) and cytokine/chemokine secretion (IFN- $\gamma$, IL-2, TNF- $\alpha$ and MIP- $1 \alpha$; figure $4 \mathrm{C}$ ) were attenuated upon co-culture of anti-VEGFR-2 CAR-T cells and target cells in the presence of VEGF-A but not VEGF-A/anti-VEGFR-2 antibody complexes or control protein.

Subsequently, we investigated whether tumor control by VEGFR-2 CAR T cells is impaired by VEGF-A in vivo. We coadministered anti-VEGF-A antibody with anti-VEGFR-2 CAR-T cells in lymphodepleted mice engrafted with B16 melanoma (illustrated in figure 4D). In this tumor model, soluble VEGF-A is highly abundant in the tumors and also present, although at much lower levels, in the serum (figure 4E). As we previously observed (figure 1D), antiVEGFR-2 CAR-T cells alone and Thy1.1-T cells failed to control tumor growth. Consistent with prior work, ${ }^{49}$ coadministration of anti-VEGF-A antibody with Thy1.1-T cells slowed tumor growth (not significant) as compared with Thy1.1-T cells alone (figure 4F), presumably by tumor vasculature normalization, and/or TME remodeling favoring endogenous immunity. However, the combination of anti-VEGF-A antibody and anti-VEGFR-2 CAR-T cells significantly controlled tumor growth in comparison to both CAR-T cells alone and Thy1.1-T cells combined with anti-VEGF-A antibody (figure 4F). Notably, mice that received the combinatorial therapy did not show signs of toxicity, undergoing similar weight changes as the other treatment groups (figure 4G). Furthermore, we observed that coadministration of anti-VEGF-A antibody and antiVEGFR-2 CAR-T cells was associated with a higher abundance of CD8 $\alpha$, IFN- $\gamma$ and CAR-T cells in the tumors in comparison to all other treatment groups as measured by RT-qPCR (figure 4H-J).

Finally, we sought to evaluate the impact of anti-VEGF-A antibody coadministration on CAR-T cell fitness in the tumor and spleen following ACT. By flow cytometric analyses, we evaluated expression of the inhibitory markers programmed cell death protein-1 (PD-1), lymphocyte activation gene 3 (LAG-3) and cytotoxic T lymphocyte associated protein-4 (CTLA-4), along with Ki67 as a marker of proliferation. We observed a lower proportion of PD- ${ }^{+}$Ki67 CAR-T cells as well as LAG- $3^{+}$Ki67 CAR-T cells in the tumors of mice treated with CAR-T cells and anti-VEGF-A antibody. In contrast, there were no significant differences in the phenotype for CAR-T cells engrafted in the spleen (figure $5 \mathrm{~A}-\mathrm{C}$ ), nor in the proportion of tumor-infiltrating CTLA- $4^{+}$Ki67 CAR-T cells (figure 5C) between the two treatment groups. Notably, we found that the combination of CAR-T cells with the anti-VEGF-A antibody did not augment VEGF-A mRNA levels in tumors as compared with CAR-T cell treatment alone (figure 5D). In summary, along with enhancing tumor control, anti-VEGF-A antibody coadministration supports CAR-T cell fitness in the TME and may alleviate exhaustion. Based on our in vitro data and abundance of VEGF-A in the TME, presumably anti-VEGF-A antibody treatment also limits competition for VEGFR-2 binding by the CAR-T cells, thereby allowing enhanced reactivity against the tumor vasculature.

\section{DISCUSSION}

It is now widely held that CAR-T cells will be insufficient as a monotherapy to control advanced solid tumors. Moreover, the identification of solid TAs that are stably, homogeneously, and broadly expressed, and that do not run the risk of on-target but off-tumor toxicity, remains elusive. As such, important research efforts are underway to identify combinatorial treatment and co-engineering strategies to promote CAR-T cell function and safety, either directly and/or via TME reprogramming to harness endogenous immunity. ${ }^{38}$ This underlies the importance of robustly evaluating CAR therapies in syngeneic tumor models that allow the interplay of the transferred murine CAR-T cells with the endogenous immune system, and the assessment of potential toxicity. ${ }^{40}$ The former effects cannot be fully evaluated in the tumors of immune compromised NOD scid gamma mouse (NSG) mice treated by human CAR-T cells, and the latter only if the scFv used to build the CAR is cross-reactive against both species.

CAR-T cell targeting of tumor vasculature antigens offers several advantages including broad expression by different solid tumor types, higher genomic stability, and accessibility of the TAs to circulating CAR-T cells. However, as in the tumor bed, a range of suppressive mechanisms can be upregulated in tumor blood vessels that attenuate T-cell function (reviewed in a previous work ${ }^{10}$ ). Several preclinical studies, including our own, have shown poor performance by 2G VEGFR-2 targeted CARs comprising the scFv DC101, unless they are provided with cytokine support (either coadministered or via co-engineering), or tumor-reactive $\mathrm{T}$ cells are co-transferred. ${ }^{29}{ }^{38}{ }^{39}$ Here we sought to identify mechanisms of suppression of tumorvasculature targeted anti-VEGFR-2 CAR-T cells in a syngeneic tumor model.

We began by evaluating VEGFR-2 expression levels but found no significant differences among CAR-T cell and control-T cell treated tumors, by both immunofluorescence and RT-qPCR. Interestingly, however, we observed 
A C Cell-expressed VEGFR-2

(1) Endothelial monolayer

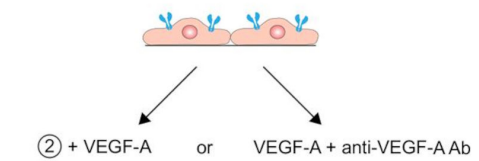

(2) + VEGF-A or VEGF-A + anti-VEGF-A Ab

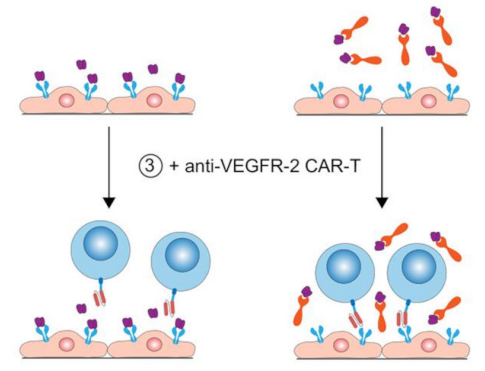

(4) Measure adhesion $(90 \mathrm{~min}) \&$ cytokines (20 hrs)
B

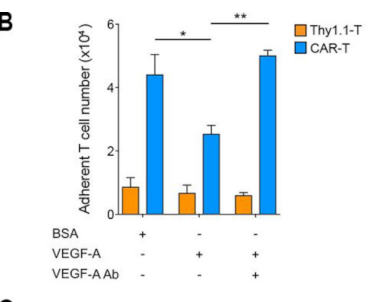

C
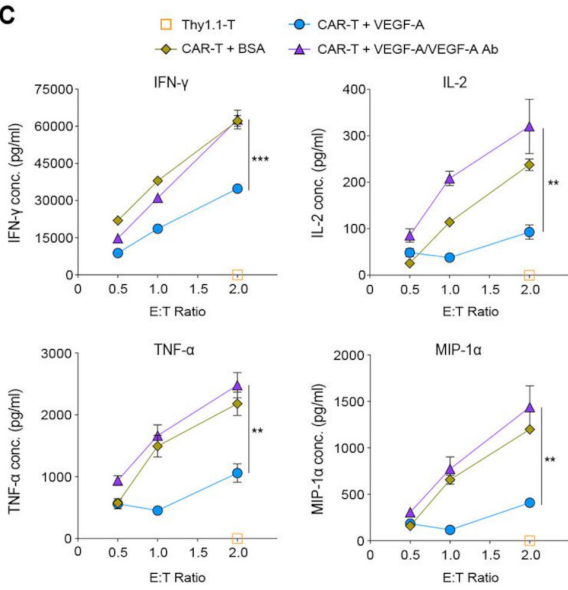

E

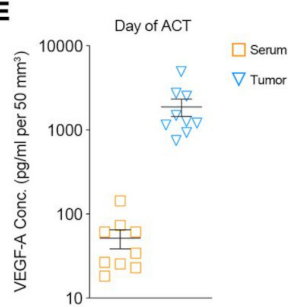

D

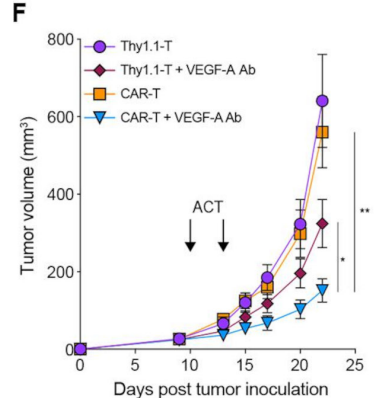

G

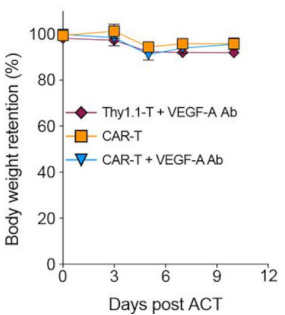

H

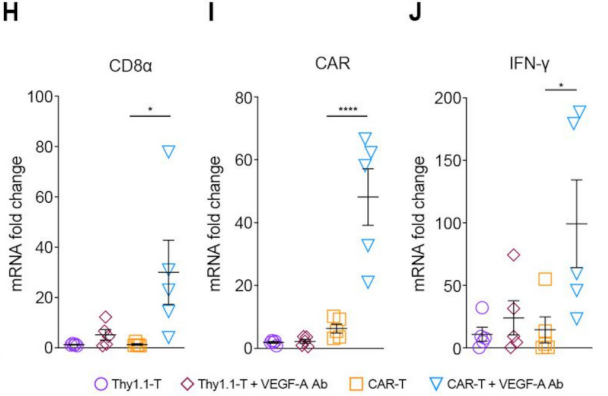

Figure 4 CAR-T cell effector function in vitro and in vivo is abrogated by soluble VEGF-A and restored in the presence of anti-VEGF-A antibody. (A) Schematic of the method used to assess the impact of soluble VEGF-A (in the presence or absence of anti-VEGF-A Ab) on the adhesion of CAR-T cells to target cells and CAR-T cell reactivity. (B) Quantification of the adhesion of Thy1.1-T cells or CAR-T cells to endothelial cells 4.5 hours post exposure to control protein, VEGF-A or VEGF-A/VEGF-A $\mathrm{Ab}$ complexes. Results are presented as the mean number of adherent cells $\pm \mathrm{SEM}$ of triplicate wells with $\mathrm{T}$ cells pooled from $\mathrm{n}=4$ mice. (C) Secretion of the indicated Th1 cytokines (IFN- $\gamma, \mathrm{IL}-2$ and TNF- $\alpha$ ) or chemokines (MIP-1 $\alpha$ ) upon co-culture of CAR-T with endothelial cells at the indicated effector:target ratios in the presence of BSA, VEGF-A or VEGF-A/VEGF-A Ab complexes (at a VEGF-A/VEGF-A Ab molar ratio of 1:3). Cytokine levels are presented as mean concentration $(\mathrm{pg} / \mathrm{mL}) \pm S E M$ of triplicate wells with T cells pooled from $n=4$ mice. (D) Schematic of ACT study and ex vivo analysis. Arrows indicate days of T-cell infusion. (E) Measurement of soluble VEGF-A levels in the serum and tumors of B16 melanoma-bearing mice at the day of ACT using ELISA. Graph presents the mean VEGF-A concentration $(\mathrm{pg} / \mathrm{ml}) \pm$ SEM normalized to tumor volume of $\mathrm{n}=9$ mice. (F) Assessment of tumor control over time for the different treatment groups. Results are expressed as mean tumor volume $\left(\mathrm{mm}^{3}{ }^{3} \mathrm{SEM}\right)$ with $\mathrm{n}=12$ mice per group pooled from two independent experiments. (G) Comparison of body weight between differently treated mice. Data show the mean percentage of body weight retention \pm SEM relative to control Thy1.1-T-treated mice with $n=12$ mice per group. (H-J) Relative quantification of mRNA for CD8 $\alpha(H)$, CAR (I), and IFN- $\gamma(J)$ in the tumors of all treatment groups. Results are presented as mean mRNA fold change (relative to Thy1.1) \pm SEM with $n=5$ mice per group. Statistical analyses in (B), (C) and (H-J) were performed using a one-way ANOVA with Tukey post hoc correction test and in (F) using a two-way repeated measures ANOVA with Newman-Keuls post hoc correction test; ${ }^{*} \mathrm{p}<0.05$; ${ }^{* *} \mathrm{p}<0.01$; ${ }^{* * *} \mathrm{p}<0.001$; ${ }^{* * * \star} \mathrm{p}<0.0001$. ACT, adoptive cell transfer; CAR, chimeric antigen receptor; IFN- $\gamma$, interferon- $\gamma$; IL-2, interleukin-2; TNF- $\alpha$, tumor necrosis factor- $\alpha$; VEGF-A, vascular endothelial growth factor-A; VEGFR-2, vascular endothelial growth factor receptor-2. 
A
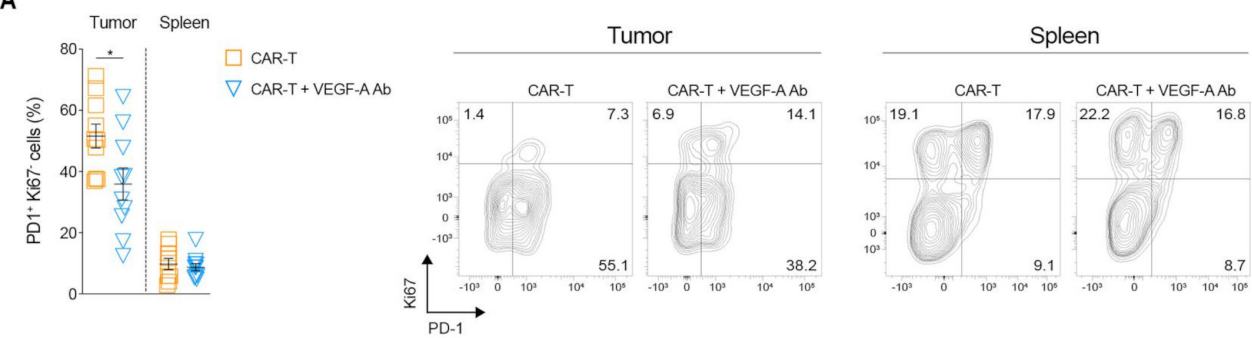

B
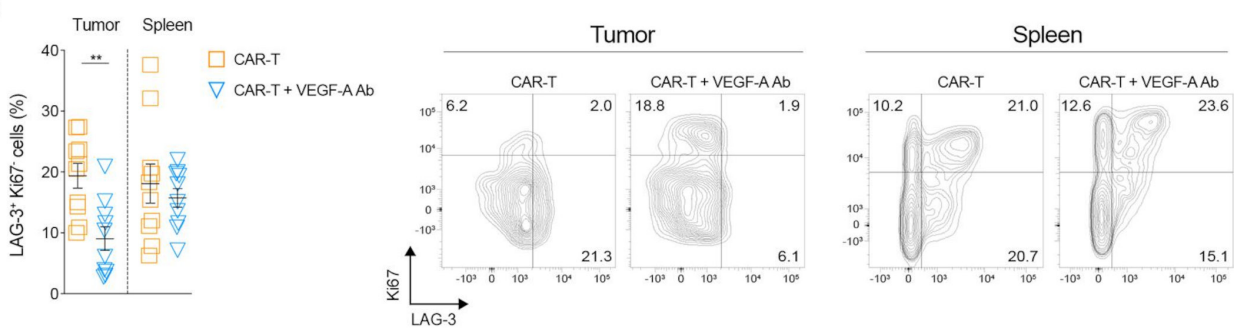

C
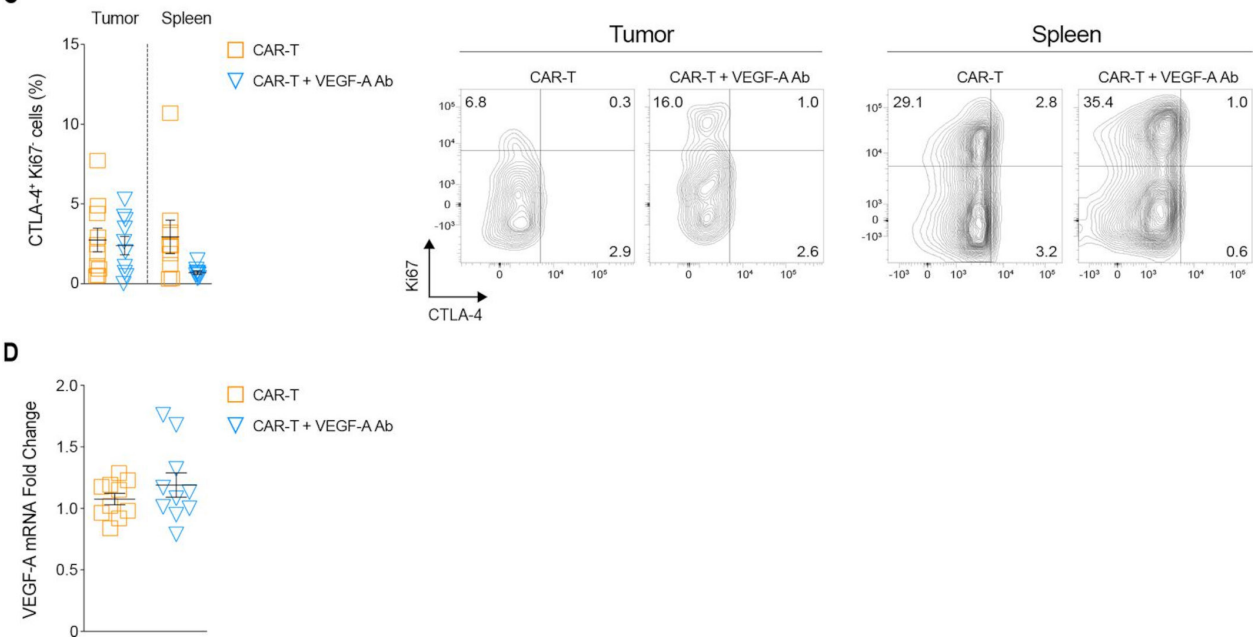

Figure 5 Coadministration of anti-VEGF-A antibody is associated with improved phenotypic fitness of tumor-infiltrating CAR-T cells. (A-C) Graph bars showing the frequency of PD-1 $1^{+}$Ki67 (A), LAG-3 ${ }^{+}$Ki67-(B), and CTLA- $4^{+}$Ki67 $(C)$ cells among CD45.1 $1^{+}$ $\mathrm{CD}^{+} \mathrm{T}$ cells in the tumors and spleens of mice treated with CAR-T cells (orange) or CAR-T cells plus anti-VEGF-A Ab (blue). (D) Relative mRNA quantification of VEGF-A in the tumors of mice treated with CAR-T cells (orange) or CAR-T cells plus antiVEGF-A Ab (blue). Results are presented as mean mRNA fold change \pm SEM with $n=10$ mice per group. Representative contour plots for each graph bar are shown on the right. All results show mean frequency $\pm S E M$ obtained from $n=10$ mice. Statistical analysis was performed using a two-tailed unpaired Student's t-test; ${ }^{*} \mathrm{p}<0.05 ;{ }^{* *} \mathrm{p}<0.01$. Ab, antibody; CAR, chimeric antigen receptor; CTLA-4, cytotoxic T lymphocyte associated protein-4; LAG-3, lymphocyte activation gene 3; PD-1, programmed cell death protein-1; VEGF-A, vascular endothelial growth factor-A.

significant gene upregulation of VEGF-A in tumors treated by CAR-T cells. Along with its potent role in driving angiogenesis, VEGF-A has been shown to cause a clustering defect of adhesion molecules and thereby limit immune cell extravasation. ${ }^{42}$ We questioned if this could be a mechanism of suppression in our model but found no evidence for alterations in ICAM- 1 or VCAM-1 (or VEGFR-2) expression levels by endothelial cells exposed to VEGF-A in vitro, despite a significant drop in adhesion by the anti-VEGFR-2 CAR-T cells. While this did not rule out clustering defects of adhesion molecules following VEGF-A exposure of the endothelial cells, we sought first to evaluate if VEGF-A, a natural angiogenic ligand of VEGFR-2, was in fact hindering engagement of the CAR itself with its target. Notably, other solid TAs including mesothelin, ${ }^{50}$ HER-2 ${ }^{52}$ and CEA ${ }^{53}$ can be shed by tumor cells but there have been no reports of CAR blockade by serum proteins. Moreover, while rituximab (an anti-CD20 monoclonal antibody) was shown to dampen the reactivity of CD20-targeted CAR-T cells in vitro, CAR-T cell control of rituximab-refractory lymphoma in vivo was not impaired by coadministration of rituximab. ${ }^{54}$

By computational modeling, we predicted an overlap in VEGFR-2 binding by VEGF-A and the DC101 scFv, and we subsequently proved by in vitro competition assays that soluble VEGF-A impaired CAR-T cell engagement with their shared target. However, VEGF-C and VEGF-D, the lower affinity ligands of VEGFR-2, ${ }^{55}$ had no impact on 
CAR-T cell binding and function. Moreover, we showed that coadministration of anti-VEGF-A antibody in vivo enhanced tumor control by anti-VEGFR-2 CAR-T cells and increased T-cell persistence. Notably, anti-VEGF-A antibody coadministration was associated with reduced frequencies of tumor-infiltrating PD- $1^{+}$Ki67 and LAG- $3^{+}$ Ki67 CAR-T cells, in line with prior research demonstrating that VEGF-A augments the expression of inhibitory checkpoints involved in $\mathrm{CD}^{+} \mathrm{T}$ cell exhaustion. ${ }^{56}$ It is possible that beyond physically sequestering VEGF-A to enable superior CAR engagement with VEGFR-2, the antibody also served to normalize vessels for enhanced extravasation of endogenous $\mathrm{T}$ cells into the tumor bed. ${ }^{42} 49758$ Moreover, VEGF-A plays a key role in generating a suppressive TME, including the accumulation of myeloid-derived suppressor cells (MDSCs), inhibition of DC maturation and induction of Tregs, ${ }^{59} 60$ and these processes may also have been blunted upon anti-VEGF-A antibody coadministration. The relative contribution of blocking the above-mentioned tumor-promoting processes by anti-VEGF-A antibody has not been addressed in this study. However, we conclude that the development of scFvs targeting other domains of VEGFR-2 that are not impaired by soluble ligand is warranted for further exploration of CAR therapy against the tumor vasculature. To our knowledge, this study is the first example of impaired function of a CAR resulting from competition with a soluble ligand. Overall, our work provides rationale for the use of combinatorial therapies that promote CAR-T cell adhesion and reprogram the TME for enhanced CAR-T cell fitness and tumor control.

\section{Author affiliations}

${ }^{1}$ Department of Oncology, Ludwig Institute for Cancer Research Lausanne, Lausanne University Hospital and University of Lausanne, Lausanne, Vaud, Switzerland

${ }^{2}$ Computer-aided Molecular Engineering Group, Department of Oncology UNIL CHUV, Ludwig Institute for Cancer Research, University of Lausanne, Lausanne, Vaud, Switzerland

Acknowledgements We kindly thank Genentech (San Francisco, California, USA) for providing anti-VEGF-A antibody (B20-4.1.1). We also wish to thank members of the Flow Cytometry Platform and the Animal Care Facility of the University of Lausanne for their support.

Contributors MI, GC and EL conceived, designed and supervised the study. MI, EL and PK wrote the manuscript. EL and PK created the figures. EL, PK, CR, EC, and GR conducted experiments, acquired and analyzed data. PR built essential constructs. VZ performed the computational protein modeling. DD reviewed the data and manuscript and provided suggestions. All authors read and approved the contents of the manuscript.

Funding This work was generously supported by Ludwig Cancer Research, an Advanced European Research Council Grant to GC (1400206AdG-322875), and the Biltema Foundation.

\section{Competing interests None declared.}

\section{Patient consent for publication Not required.}

Ethics approval All in vivo experiments were conducted in accordance with approval from the Service of Consumer and Veterinary Affairs (SCAV) of the Canton of Vaud.

Provenance and peer review Not commissioned; externally peer reviewed.
Data availability statement All data relevant to the study are included in the article or uploaded as supplementary information. Data sharing not applicable as no datasets generated and/or analyzed for this study.

Supplemental material This content has been supplied by the author(s). It has not been vetted by BMJ Publishing Group Limited (BMJ) and may not have been peer-reviewed. Any opinions or recommendations discussed are solely those of the author(s) and are not endorsed by BMJ. BMJ disclaims all liability and responsibility arising from any reliance placed on the content. Where the content includes any translated material, BMJ does not warrant the accuracy and reliability of the translations (including but not limited to local regulations, clinical guidelines, terminology, drug names and drug dosages), and is not responsible for any error and/or omissions arising from translation and adaptation or otherwise.

Open access This is an open access article distributed in accordance with the Creative Commons Attribution Non Commercial (CC BY-NC 4.0) license, which permits others to distribute, remix, adapt, build upon this work non-commercially, and license their derivative works on different terms, provided the original work is properly cited, appropriate credit is given, any changes made indicated, and the use is non-commercial. See http://creativecommons.org/licenses/by-nc/4.0/.

\section{ORCID iDs}

Evripidis Lanitis http://orcid.org/0000-0002-8194-5414

Paris Kosti http://orcid.org/0000-0002-8558-1064

Melita Irving http://orcid.org/0000-0002-6849-7194

\section{REFERENCES}

1 Neelapu SS, Locke FL, Bartlett NL, et al. Axicabtagene Ciloleucel CAR T-cell therapy in refractory large B-cell lymphoma. N Engl J Med 2017;377:2531-44.

2 Schuster SJ, Svoboda J, Chong EA, et al. Chimeric antigen receptor T cells in refractory $\mathrm{B}-$ cell lymphomas. $N$ Engl J Med 2017;377:2545-54.

3 Lanitis E, Coukos G, Irving M. All systems go: converging synthetic biology and combinatorial treatment for CAR-T cell therapy. Curr Opin Biotechnol 2020;65:75-87.

4 Brown CE, Alizadeh D, Starr R, et al. Regression of glioblastoma after chimeric antigen receptor T-cell therapy. $N$ Engl J Med 2016;375:2561-9.

5 Morgan RA, Yang JC, Kitano M, et al. Case report of a serious adverse event following the administration of $\mathrm{T}$ cells transduced with a chimeric antigen receptor recognizing ErbB2. Mol Ther 2010;18:843-51.

6 Leko V, Rosenberg SA. Identifying and targeting human tumor antigens for $\mathrm{T}$ cell-based immunotherapy of solid tumors. Cancer Cell 2020;38:454-72.

7 Kosti P, Maher J, Arnold JN. Perspectives on chimeric antigen receptor T-cell immunotherapy for solid tumors. Front Immunol 2018;9:1104.

8 Lanitis E, Dangaj D, Irving M, et al. Mechanisms regulating T-cell infiltration and activity in solid tumors. Ann Oncol 2017;28:xii18-32.

9 Irving M, Vuillefroy de Silly R, Scholten K, et al. Engineering Chimeric Antigen Receptor T-Cells for Racing in Solid Tumors: Don't Forget the Fuel. Front Immunol 2017;8:267.

10 Lanitis E, Irving M, Coukos G. Targeting the tumor vasculature to enhance T cell activity. Curr Opin Immunol 2015;33:55-63.

11 Hanahan D, Folkman J. Patterns and emerging mechanisms of the angiogenic switch during tumorigenesis. Cell 1996;86:353-64.

12 Folkman J. Angiogenesis in cancer, vascular, rheumatoid and other disease. Nat Med 1995;1:27-30.

13 Ferrara N, Gerber H-P, LeCouter J. The biology of VEGF and its receptors. Nat Med 2003;9:669-76.

14 Nowak-Sliwinska P, Alitalo K, Allen E, et al. Consensus guidelines for the use and interpretation of angiogenesis assays. Angiogenesis 2018;21:425-532.

15 Ramjiawan RR, Griffioen AW, Duda DG. Anti-Angiogenesis for cancer revisited: is there a role for combinations with immunotherapy? Angiogenesis 2017;20:185-204.

16 van Beijnum JR, Nowak-Sliwinska P, Huijbers EJM, et al. The great escape; the hallmarks of resistance to antiangiogenic therapy. Pharmacol Rev 2015;67:441-61.

17 Bergers G, Hanahan D. Modes of resistance to anti-angiogenic therapy. Nat Rev Cancer 2008;8:592-603.

18 Jain RK. Normalization of tumor vasculature: an emerging concept in antiangiogenic therapy. Science 2005;307:58-62.

19 Thorpe PE. Vascular targeting agents as cancer therapeutics. Clin Cancer Res 2004;10:415-27. 
20 Akbari P, Huijbers EJM, Themeli M, et al. The tumor vasculature an attractive CAR T cell target in solid tumors. Angiogenesis 2019;22:473-5.

21 O'Rourke DM, Nasrallah MP, Desai A, et al. A single dose of peripherally infused EGFRvIll-directed CAR T cells mediates antigen loss and induces adaptive resistance in patients with recurrent glioblastoma. Sci Trans/ Med 2017;9:eaaa0984. doi:10.1126/ scitransImed.aaa0984

22 Majzner RG, Mackall CL. Tumor antigen escape from CAR T-cell therapy. Cancer Discov 2018;8:1219-26.

23 Boehm T, Folkman J, Browder T, et al. Antiangiogenic therapy of experimental cancer does not induce acquired drug resistance. Nature 1997;390:404-7.

24 Kalos M, Levine BL, Porter DL, et al. T cells with chimeric antigen receptors have potent antitumor effects and can establish memory in patients with advanced leukemia. Sci Trans/ Med 2011;3:95ra73.

25 Kuhn NF, Purdon TJ, van Leeuwen DG, et al. Cd40 Ligand-Modified chimeric antigen receptor $T$ cells enhance antitumor function by eliciting an endogenous antitumor response. Cancer Cell 2019;35:473-88.

26 Wang W, Ma Y, Li J, et al. Specificity redirection by CAR with human VEGFR-1 affinity endows T lymphocytes with tumor-killing ability and anti-angiogenic potency. Gene Ther 2013;20:970-8.

27 Inoo K, Inagaki R, Fujiwara K, et al. Immunological quality and performance of tumor vessel-targeting CAR-T cells prepared by mRNA-EP for clinical research. Mol Ther Oncolytics 2016:3:16024.

28 Kanagawa N, Yanagawa T, Nakagawa T, et al. Tumor vessel-injuring ability improves antitumor effect of cytotoxic T lymphocytes in adoptive immunotherapy. Cancer Gene Ther 2013;20:57-64.

29 Chinnasamy D, Yu Z, Theoret MR, et al. Gene therapy using genetically modified lymphocytes targeting VEGFR-2 inhibits the growth of vascularized syngenic tumors in mice. J Clin Invest 2010;120:3953-68.

30 Kulemzin SV, Gorchakov AA, Chikaev AN, et al. VEGFR2-specific FnCAR effectively redirects the cytotoxic activity of T cells and YT NK cells. Oncotarget 2018:9:9021-9.

31 Hajari Taheri F, Hassani M, Sharifzadeh Z, et al. T cell engineered with a novel nanobody-based chimeric antigen receptor against VEGFR2 as a candidate for tumor immunotherapy. IUBMB Life 2019;71:1259-67.

32 Niederman TMJ, Ghogawala Z, Carter BS, et al. Antitumor activity of cytotoxic $T$ lymphocytes engineered to target vascular endothelial growth factor receptors. Proc Natl Acad Sci U S A 2002;99:7009-14

33 Fu X, Rivera A, Tao L, et al. Genetically modified T cells targeting neovasculature efficiently destroy tumor blood vessels, shrink established solid tumors and increase nanoparticle delivery. Int $J$ Cancer 2013;133:2483-92.

34 Wagner J, Wickman E, Shaw TI, et al. Antitumor effects of CAR $T$ cells redirected to the EDB splice variant of fibronectin. Cancer Immunol Res 2021;9:279-90.

35 Zhang T, Sentman CL. Mouse tumor vasculature expresses NKG2D ligands and can be targeted by chimeric NKG2D-modified T cells. $J$ Immunol 2013;190:2455-63.

36 Santoro SP, Kim S, Motz GT, et al. T cells bearing a chimeric antigen receptor against prostate-specific membrane antigen mediate vascular disruption and result in tumor regression. Cancer Immunol Res 2015;3:68-84.

37 Byrd TT, Fousek K, Pignata A, et al. TEM8/ANTXR1-Specific CAR T cells as a targeted therapy for triple-negative breast cancer. Cancer Res 2018;78:489-500.

38 Chinnasamy D, Tran E, Yu Z, et al. Simultaneous targeting of tumor antigens and the tumor vasculature using $T$ lymphocyte transfer synergize to induce regression of established tumors in mice. Cancer Res 2013;73:3371-80.

39 Chinnasamy D, Yu Z, Kerkar SP, et al. Local delivery of interleukin-12 using $T$ cells targeting VEGF receptor- 2 eradicates multiple vascularized tumors in mice. Clin Cancer Res 2012;18:1672-83.
40 Lanitis E, Rota G, Kosti P, et al. Optimized gene engineering of murine CAR-T cells reveals the beneficial effects of IL-15 coexpression. J Exp Med 2021;218:e20192203.

41 Motz GT, Santoro SP, Wang L-P, et al. Tumor endothelium FasL establishes a selective immune barrier promoting tolerance in tumors. Nat Med 2014;20:607-15.

42 Bouzin C, Brouet A, De Vriese J, et al. Effects of vascular endothelial growth factor on the lymphocyte-endothelium interactions: identification of caveolin-1 and nitric oxide as control points of endothelial cell anergy. J Immunol 2007;178:1505-11.

43 Franklin MC, Navarro EC, Wang Y, et al. The structural basis for the function of two anti-VEGF receptor 2 antibodies. Structure 2011;19:1097-107.

44 Edgar RC. Muscle: a multiple sequence alignment method with reduced time and space complexity. BMC Bioinformatics 2004;5:113.

45 Webb B, Sali A. Protein structure modeling with MODELLER. Methods Mol Biol 2017;1654:39-54.

46 Shen M-Y, Sali A. Statistical potential for assessment and prediction of protein structures. Protein Sci 2006;15:2507-24.

47 Pettersen EF, Goddard TD, Huang CC, et al. UCSF Chimera--a visualization system for exploratory research and analysis. J Comput Chem 2004;25:1605-12.

48 Brozzo MS, Bjelić S, Kisko K, et al. Thermodynamic and structural description of allosterically regulated VEGFR-2 dimerization. Blood 2012;119:1781-8.

49 Shrimali RK, Yu Z, Theoret MR, et al. Antiangiogenic agents can increase lymphocyte infiltration into tumor and enhance the effectiveness of adoptive immunotherapy of cancer. Cancer Res 2010;70:6171-80.

50 Lanitis E, Poussin M, Hagemann IS, et al. Redirected antitumor activity of primary human lymphocytes transduced with a fully human anti-mesothelin chimeric receptor. Mol Ther 2012;20:633-43.

51 Adusumilli PS, Cherkassky L, Villena-Vargas J, et al. Regional delivery of mesothelin-targeted CAR T cell therapy generates potent and long-lasting CD4-dependent tumor immunity. Sci Transl Med 2014:6:261ra151.

52 Beecham EJ, Ortiz-Pujols S, Junghans RP. Dynamics of tumor cell killing by human $T$ lymphocytes armed with an anti-carcinoembryonic antigen chimeric immunoglobulin T-cell receptor. J Immunother 2000;23:332-43.

53 Nolan KF, Yun CO, Akamatsu Y. Bypassing immunization: optimized design of "designer T cells" against carcinoembryonic antigen (CEA)expressing tumors, and lack of suppression by soluble CEA. Clin Cancer Res 1999;5:3928-41.

54 Rufener GA, Press OW, Olsen P, et al. Preserved activity of CD20Specific chimeric antigen receptor-expressing T cells in the presence of rituximab. Cancer Immunol Res 2016;4:509-19.

55 King C, Hristova K. Direct measurements of VEGF-VEGFR2 binding affinities reveal the coupling between ligand binding and receptor dimerization. J Biol Chem 2019;294:9064-75.

56 Voron T, Colussi O, Marcheteau E, et al. Vegf-A modulates expression of inhibitory checkpoints on CD8+ T cells in tumors. $J$ Exp Med 2015;212:139-48.

57 Wong P-P, Bodrug N, Hodivala-Dilke KM. Exploring novel methods for modulating tumor blood vessels in cancer treatment. Curr Biol 2016;26:R1161-6.

58 Bocca P, Di Carlo E, Caruana I, et al. Bevacizumab-mediated tumor vasculature remodelling improves tumor infiltration and antitumor efficacy of GD2-CAR T cells in a human neuroblastoma preclinical model. Oncoimmunology 2017;7:e1378843.

59 Gabrilovich DI, Chen HL, Girgis KR, et al. Production of vascular endothelial growth factor by human tumors inhibits the functional maturation of dendritic cells. Nat Med 1996;2:1096-103.

60 Huang Y, Chen X, Dikov MM, et al. Distinct roles of VEGFR-1 and VEGFR-2 in the aberrant hematopoiesis associated with elevated levels of VEGF. Blood 2007:110:624-31. 